\title{
Silicon Nanowire Microfluidic Biosensor for Multiplexed Biomolecule Detection
}

\author{
Anran Gao, Yuelin Wang, and Tie $\mathrm{Li}^{*}$ \\ Science and Technology on Micro-system Laboratory, Shanghai Institute of Microsystem \\ and Information Technology, Chinese Academy of Sciences, Shanghai 200050, China
}

(Received May 25, 2018; accepted September 10, 2018)

Keywords: silicon nanowire, microfluidic system, multiplexed detection, biosensor

The integration of a microfluidic sample delivery chip with a silicon nanowire (SiNW) biosensor device for multiplexed biomolecule detection was demonstrated. The SiNWs and polydimethylsiloxane (PDMS) chips were fabricated by complementary metal oxide semiconductor (CMOS) compatibility methods at a low cost. They were integrated together by using optimal $\mathrm{O}_{2}$ plasma parameters that enabled rapid and leakage-free bond formation, without additional heating or applied pressure. The capillary action induced by the hydrophilicity of the channels using polyvinylpyrrolidone (PVP) was demonstrated to allow analyte solution delivery onto the sensor array directly, without the need of using external pumping devices. The multiplexed, real-time, and label-free electrical biomolecule detection was demonstrated with high sensitivity and selectivity. The novel detection approach enables simple and rapid multiplexed biomarker detection, showing great promise for application in point-of-care disease diagnostics.

\section{Introduction}

The ultrasensitive detection of biological and chemical species is critical in many areas, including disease diagnosis, drug discovery, and biomolecular analysis. ${ }^{(1-3)}$ Silicon nanowire (SiNW) field-effect transistors (FETs) with a small size and a high surface-to-volume ratio are emerging as powerful sensors for recognizing a wide range of biological and chemical species, including DNA, proteins, small molecules, metal ions, single virus particles, and even cells. ${ }^{(1,4-9)}$ The multiplexed detection of multiple biomolecular targets with high sensitivity and specificity is particularly important. However, a previous work that showed the capability for the selective multiplexed detection of different molecules typically used a spotting robot for localization, facing limits of high price and limited availability. ${ }^{(1)}$

The integration of a microfluidic channel system with a SiNW device can provide a cheap and simple method for multiplexed detection. ${ }^{(10,11)}$ Furthermore, the analyte solution delivery system can be used to guide the sample flow directly on the sensing surface to reduce the loss of possibly expensive and limited samples. A crucial step in the integration of the sample delivery

*Corresponding author: e-mail: tli@mail.sim.ac.cn

https://doi.org/10.18494/SAM.2018.2007 
system is to provide a leakage-free seal between the microfluidic chip and the sensor device. However, the use of pristine polymer channels for sample delivery may lead to extensive bubble formation and the nonspecific adsorption of sample molecules onto the channel surfaces. ${ }^{(12,13)}$ However, to date, the system used to deliver the analyte solution as an important factor in biomolecule detection has not yet been the focus of any studies.

In this work, we studied the method of integrating a hydrophilic and leakage-free polydimethylsiloxane (PDMS) sample delivery chip onto a SiNW device, and we demonstrated its application in multiplexed biomolecule detection. SiNWs with complementary metal oxide semiconductor (CMOS) compatibility as well as low cost were fabricated by anisotropic selfstop etching on silicon-on-insulator (SOI) wafers. PDMS chips with channels were fabricated using silicon ( $\mathrm{Si}$ ) molds by rapid, simple, and cost-effective fabrication techniques. The PDMS chips were bonded onto the SiNW sensor surfaces by using optimal $\mathrm{O}_{2}$ plasma parameters that enabled rapid bond formation, without additional heating or applied pressure. In addition, biocompatible and hydrophilic polyvinylpyrrolidone (PVP) was used for the formation of a longterm hydrophilic coating on channel surfaces, to enable capillary action, and thus to exclude the use of external pumping devices in sample delivery. By using the integrated SiNW-PDMS device, the highly sensitive, highly selective, label-free and multiplexed electrical detection of biomolecules was demonstrated, resulting in a portable device with great potential for disease diagnostics.

\section{Materials and Methods}

\subsection{Materials and reagents}

Sylgard 184 PDMS was purchased from Dow Corning (USA). PVP powder was purchased from Merck MGaA (Germany). 3-Aminopropyltriethoxysilane (APTES), bovine serum albumin (BSA), and glutaraldehyde were purchased from Sigma-Aldrich (USA) and used as received. Cyfra21-1 was purchased from Fitzgerald (Acton, MA, USA). Monoclonal antibody for Cyfra21-1 was purchased from US Biological Inc. (Swampscott, MA, USA). The probe DNA sample was custom-synthesized and purified by Shanghai Sangon Bioengineering Technology and Services Co., Ltd. (Shanghai, China). The microRNA (miRNA) sequences (miRNA-125: 5'-UCCCUGAGACCCUUUAACCUGUGA-3'; miRNA-126: 5'-UCGUACCGUGAGUAAUAAUGCG-3') were synthesized and purified by Life Technologies Co. (Shanghai, China). Other chemicals were obtained from Sinopharm Chemical Reagent Co., Ltd. (Shanghai, China). Solutions were prepared with Milli-Q deionized (DI) water (18 $\mathrm{M} \Omega \mathrm{cm}$, Millipore) from a Millipore system.

\subsection{Fabrication of SiNW biosensor}

The microfabrication process for our SiNW-FETs is described in detail in our previous papers. ${ }^{(14,15)}$ For the device fabrication, we started from a 4-inch (100)-oriented SIMOX (SOI) wafer, with light boron adulteration of $5 \times 10^{15} \mathrm{~cm}^{-3}$. After the formation of a patterned $\mathrm{SiO}_{2}$ 
mask on the wafer, Si was etched off by using tetramethyl ammonium hydroxide (TMAH, 25 $\mathrm{wt} \%, 50^{\circ} \mathrm{C}$ ). By employing the TMAH that etches Si (111) planes about 100 times more slowly than all other planes, the boundary of the top silicon layer was automatically aligned to the $<110>$ direction, and a very smooth (111) plane could be formed beneath the $\mathrm{SiO}_{2}$ mask. Then, a thin nitride film was deposited to protect the (111) plane previously defined. After the nitride film was patterned by ion beam etching, the $100 \mathrm{~nm} \mathrm{SiO}$ layer beneath was totally removed by buffered oxide etching (BOE). Then, by using TMAH for the second time, a controllable SiNW with a triangular cross section was finally obtained.

\subsection{Fabrication of microfluidic chip}

The fabrication of a microfluidic sample delivery chip consisted of two steps: master mold fabrication and PDMS replicas. The fabrication of the master mold started from a 4 inch $\mathrm{Si}$ wafer. First, a 1- $\mu \mathrm{m}$-thick silicon dioxide layer was thermally grown on the $\mathrm{Si}$ wafer. After then, the wafer was patterned by photolithography and the $\mathrm{SiO}_{2}$ layer was etched by BOE. Then, silicon was etched by deep reactive-ion etching (RIE) with the oxide layer and a photoresist as the protective layer. Finally, the protective layer was removed and the silicon mold was finished. For PDMS replicas, the Sylgard 184 base polymer and curing agent (10:1, w/w) were firstly thoroughly mixed and then poured onto the master mold to produce an $\sim 1.5-\mathrm{mm}$ thick layer. After that, the air trapped in the liquid PDMS was removed. Finally, the liquid PDMS was cured at $80{ }^{\circ} \mathrm{C}$ for $1 \mathrm{~h}$. The cured PDMS elastomer was cut into pieces and the liquid inlets and outlets were produced.

\subsection{Channel coating of PDMS chip}

A $1 \mathrm{mM}$ PVP solution in DI water was prepared. The channels were filled with the prepared PVP solution for at least $5 \mathrm{~min}$ at room temperature. Then, the channels were rinsed with DI water and dried with nitrogen flow.

\subsection{Bonding of SiNW device and PDMS chip}

Before $\mathrm{O}_{2}$ plasma treatment, both the SiNW device and the PDMS chip were rinsed with ethanol and DI water, and dried with nitrogen. The optimal parameters (exposure time $30 \mathrm{~s}$, $\mathrm{O}_{2}$ flow rate $500 \mathrm{ml} / \mathrm{min}$, and $\mathrm{RF}$ power $50 \mathrm{~W}$ ) were used for surface treatment using an MVD 100 system [Applied Microstructures Inc. (USA)]. After the activation, the PDMS chip was immediately placed and aligned onto the SiNW device surface using a microscope.

\subsection{Surface modification}

The SiNW array was first cleaned in a piranha solution at $90{ }^{\circ} \mathrm{C}$ in order to clean the sample surfaces as well as to generate more hydrophilic surfaces. Then, it was functionalized by exposing the surface to an APTES solution (2\% ethanol) overnight, followed by rinsing with 
absolute ethanol to remove the unreacted APTES. After that, a self-assembled monolayer with a terminal amino group was prepared by heating at $120{ }^{\circ} \mathrm{C}$ for $5 \mathrm{~min}$. A biofunctional linker, glutaraldehyde, was then covalently attached onto the amine-terminated surface by immersing the chip in a solution of $2.5 \%$ glutaraldehyde in a buffer solution $(20 \mathrm{mM} \mathrm{NaCl}, 10$ $\mathrm{mM}$ phosphate, $\mathrm{pH} 7.4$ ) at $37{ }^{\circ} \mathrm{C}$ for $1 \mathrm{~h}$. Antibody/probe attachment onto the SiNW surface was carried out by exposing the SiNWs to $50 \mu \mathrm{g} / \mathrm{ml}$ antibody of Cyfra21-1 or $1 \mu \mathrm{M}$ probe DNA in a buffer solution at $37^{\circ} \mathrm{C}$ for $2 \mathrm{~h}$ and then thoroughly washing them with the same buffer. To prevent the nonspecific binding of proteins in the detection step, the unreacted aldehydic groups were passivated by adding $10 \mathrm{mM}$ BSA solution onto the SiNW surface and incubated at $37{ }^{\circ} \mathrm{C}$ for $1 \mathrm{~h}$, followed by washing with the same buffer.

\subsection{Sensing apparatus and parameters}

For biomolecule detection, the measurements were performed using a Keithley 4200 semiconductor parameter analyzer (Keithley Instruments Inc., Cleveland, OH). The solutions used in hybridization studies were $0.01 \times$ PBS for Cyfra21-1 and 0.1× PBS for miRNA, in which the Debye length is sufficiently large to ensure effective sensing during the capture process. The current through the SiNW device was monitored while immersed in a buffer solution to establish the baseline current, relative to which changes in current would be measured. Each measurement was carried out on a separate device.

\section{Results and Discussion}

\subsection{Fabricated SiNW device and PDMS chip}

The SiNWs were fabricated by using a CMOS-compatible anisotropic self-stop etching technique that yields nanowires with dominant Si (111) exposed planes. As illustrated in Fig. 1(a), the SiNWs are grouped in clusters (10 wires each) with two nanowire arrays. The zoomed-in optical microscopy image [Fig. 1(b)] and scanning electron microscopy (SEM) image [Fig. 1(c)] show typical aspects of the fabricated nanowires. Owing to the planarization of the anisotropic wet etching, the SiNW surface was fairly smooth. The PDMS chip containing microchannels was fabricated by applying liquid PDMS over a premade silicon mold and partially cured at $80^{\circ} \mathrm{C}$. The typical microfluidic sample delivery chip is shown in Fig. 1(d).

\subsection{Integration of SiNW device and PDMS chip}

SiNW chips and PDMS samples were surface-activated as described in the experiment section and brought into contact immediately after the plasma treatment. The final integrated chip bonded on a printed circuit board (PCB) is shown in top and cross-sectional views in Figs. 2(a) and 2(b), respectively. The microfluidic devices are placed on top of the nanosensor so that the solution can be directed over the SiNWs. The bonding was observed to happen in less than 3 min. A photograph of a biosensor chip on a PCB with an integrated sample delivery system 


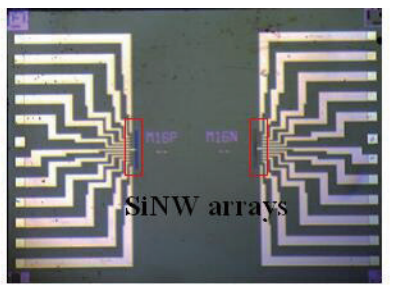

(a)

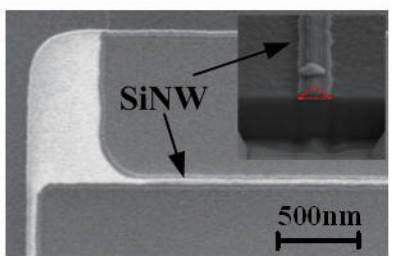

(c)

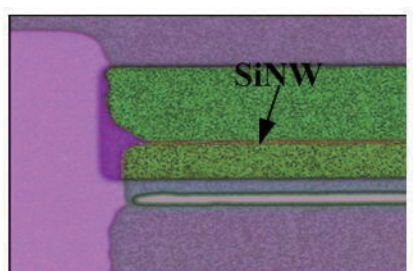

(b)

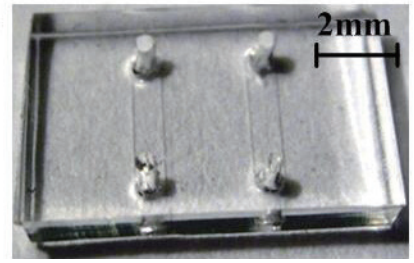

(d)

Fig. 1. (Color online) (a) Picture of a completed SiNW device. (b) Optical microscopy image of a SiNW. (c) SEM image of a nanowire in SiNW arrays. (d) PDMS sample delivery chip with two $800-\mu \mathrm{m}-$ wide and $100-\mu \mathrm{m}-\mathrm{high}$ channels.

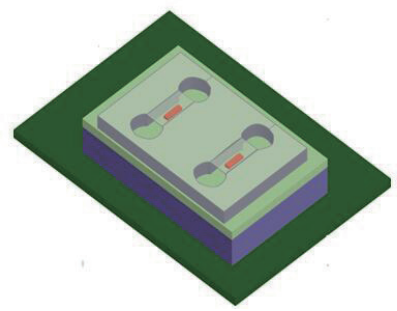

(a)

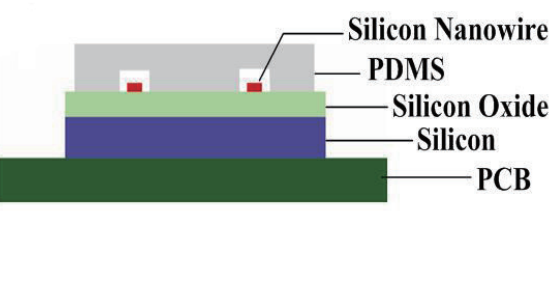

(b)

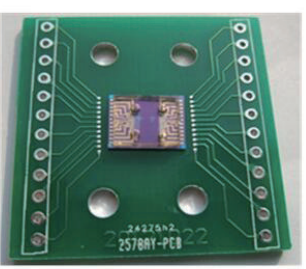

(c)

Fig. 2. (Color online) Schematic diagram of a completed SiNW device integrated with a PDMS chip in (a) top and (b) cross-sectional views. (c) Integrated PDMS-SiNW device with two sample delivery channels.

is shown in Fig. 2(c). The tearing test for the sample bonding demonstrated that an irreversible bond was formed between the PDMS chip and the SiNW device.

The $\mathrm{O}_{2}$ plasma treatment can change the surface states of both PDMS and SiNW chips, which were characterized by the sessile drop method. Contact angle graphs for the $\mathrm{O}_{2}$-plasmaactivated PDMS are presented in Fig. 3 (red), where the average values of three parallel samples with standard deviations were included. The $\mathrm{O}_{2}$-activated PDMS surfaces started to recover their hydrophobicities immediately following the treatment, reaching almost the original contact angle within several days. This is assumed to be due to the penetration of uncured low-molecular-weight species from the bulk PDMS to the surface. A polymer coating was synthesized on microchannel surfaces to prevent the hydrophobicity recovery of the $\mathrm{O}_{2}$-plasmaactivated PDMS surfaces. PVP molecules were grafted to the oxygen radicals produced during the $\mathrm{O}_{2}$ plasma activation of the PDMS surface, which kept the surface hydrophilicity (Fig. 3, black). Therefore, the capillary-action-based sample delivery was realized by the improved wetting properties. The capillary action allows the user to inject the desired sample volume onto the inlet of the delivery systems using a pipette, which excludes the need for external 


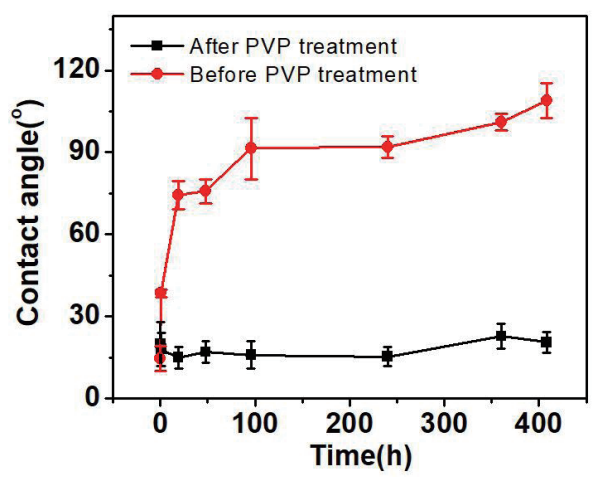

Fig. 3. (Color online) Average contact angles with standard deviations as a function of aging time for $\mathrm{O}_{2}$-plasmaactivated and PVP-treated PDMS samples.

pumping devices. Furthermore, the PVP treatment can prevent the nonspecific adsorption of sample molecules onto the channel surfaces.

\subsection{Multiplexed biomolecule detection}

In this study, silicon nanowire biosensors for multiplexed biomolecule detection were realized by immobilizing specific antibodies and probe DNA onto the SiNWs surface in separate channels. The details are shown in Fig. 4. The SiNW surface with oxide coating on it was firstly modified with conventional silane compound, APTES, as described above, generating a monolayer with a terminal amine group. The glutaraldehyde was then modified on the SiNW surface, followed by the covalent attachment of probe molecules. Then, the freshly prepared SiNW chips were subjected immediately to multiplexed molecule electrical testing. The presence of charged species on the surface of an active nanowire induces a fractional change in nanowire conductance and enables biomolecule detection. ${ }^{(16,17)}$ Cyfra21-1 and miRNA-126, which have been proven to be sensitive biomarkers in the diagnosis and prognosis of lung cancer, ${ }^{(18-20)}$ were selected as analytes. The multiplexed detection of the biomarkers may be helpful in indicating the state of lung cancer to greatly improve its early diagnosis and prognosis. ${ }^{(21)}$

For antibody-Cyfra21-1 modified SiNWs, the real-time electrical detection is demonstrated in Fig. 5(a) by delivering a series of concentrations of Cyfra21-1 onto the SiNW surface sequentially. The $0.01 \times$ PBS buffer was added on the nanowire device to establish a baseline current. After a stable reading was obtained, known concentrations of Cyfra21-1 were introduced to obtain the current change relative to the baseline current. Cyfra21-1 is negatively charged at $\mathrm{pH} 7.4$ with an isoelectric point (pI) of 5.245. ${ }^{(22)}$ Thus, as expected, the current decreased upon the binding of CYFRA21-1 to the antibody receptor on an n-type SiNW device. Figure 5(a) shows that the current change increased with increasing target concentration. The signal continues to change for CYFRA21-1 of lower concentrations, probably owing to continued diffusion and binding to the device. The nanosensor could reliably detect Cyfra21-1 at a concentration of as low as $1 \mathrm{fg} / \mathrm{ml}$. As a control experiment, $1 \mathrm{pg} / \mathrm{ml}$ carcinoembryonic 


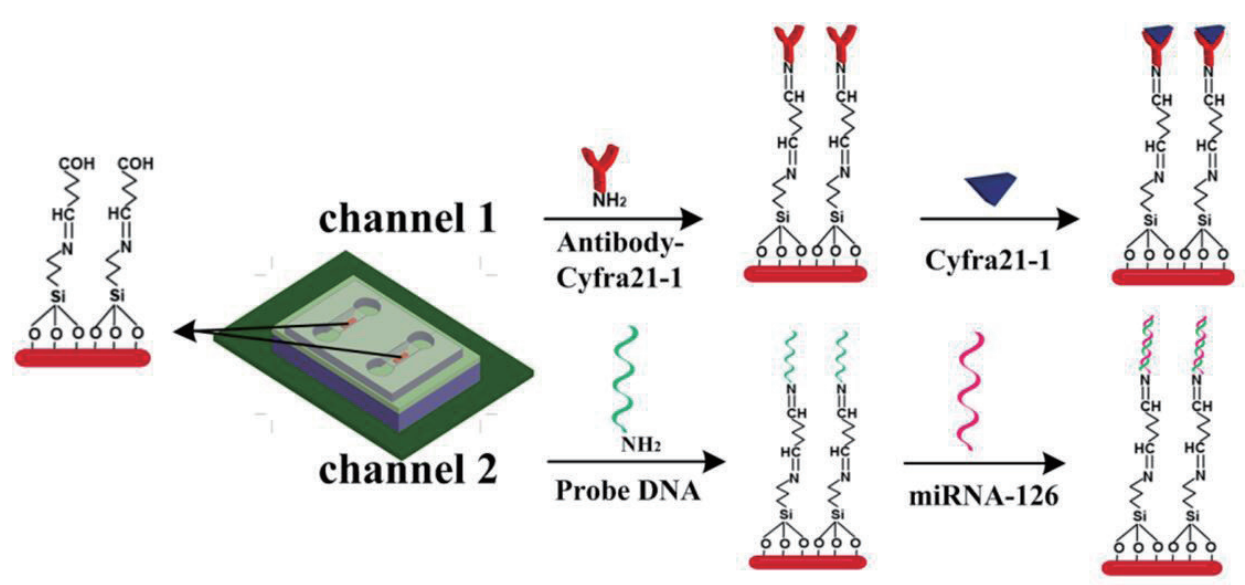

Fig. 4. (Color online) Schematic illustration of the chemical process for multiplexed and label-free electrical detection of biomolecules.

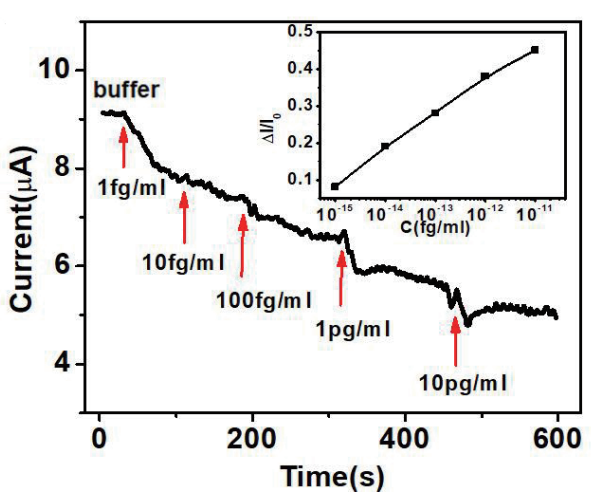

(a)

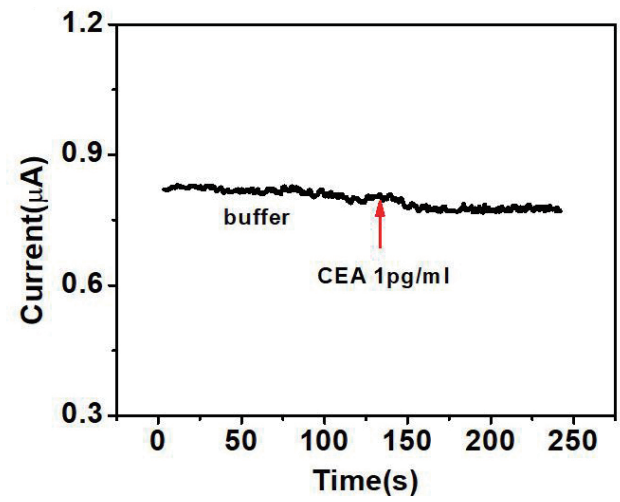

(b)

Fig. 5. (Color online) (a) Plot of real time current vs time when solutions with a series of concentrations of Cyfra21-1 were sequentially delivered onto an antibody-Cyfra21-1-modified SiNW surface. The arrows mark the points when the solutions were introduced. (b) Plot of SiNW current vs time of an antibody-Cyfra21-1-modified SiNW for real-time detection of CEA, showing high specificity of the device.

antigen (CEA) was allowed to flow through a modied SiNW-FET, which did not lead to a significant current change [Fig. 5(b)], suggesting the absence of nonspecific binding onto the SiNW surface.

An exploration of the detection limit of the probe-DNA-modified SiNW is also demonstrated in Fig. 6(a). For the n-type NW-FET device, when the negatively charged molecule miRNA-126 binds onto the SiNW surface, the carriers in the nanowire are depleted, leading to a decrease in the current of the FET device. The hybridization process could be monitored in real time using this SiNW-FET sensor. The decrease in electrical current suggests the binding of a negatively charged target onto the SiNW-FET. The detection limit of the nanosensor decreases to 0.1 fM. The selectivity of the nanosensor was investigated with miRNA-125 [Fig. 6(b)]. When miRNA-125 was allowed to flow through the sensor surface, there was essentially no significant change in electrical current, demonstrating the high selectivity of the device. Furthermore, the measurements were repeated in multiple samples and the results were consistent. 


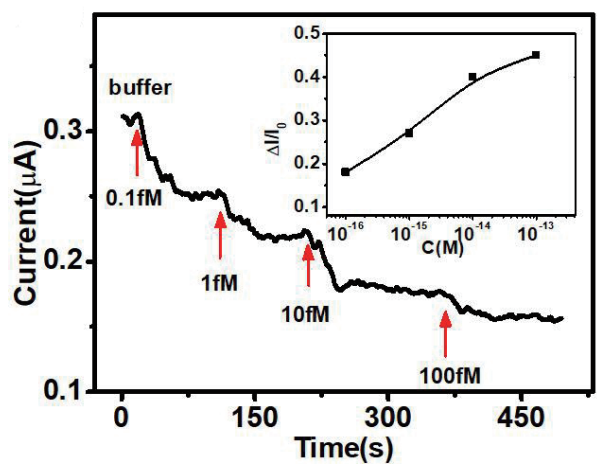

(a)

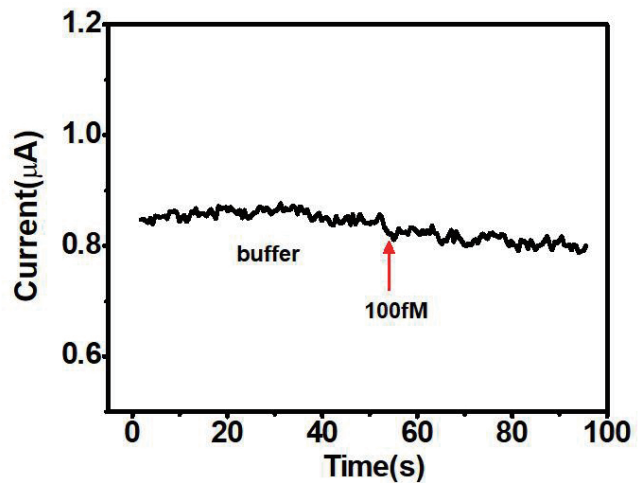

(b)

Fig. 6. (Color online) (a) Plot of real-time current vs time when solutions with a series of concentrations of miRNA-126 were sequentially delivered onto the probe-DNA-modified SiNW surface. The arrows mark the points when the solutions were introduced. (b) Plot of SiNW current vs time of probe-modified SiNW for real-time detection of miRNA-125.

\section{Conclusions}

In this study, we introduced a technique for the integration of a PDMS sample delivery chip onto a SiNW-based biosensor device and demonstrated multiplexed biomolecule detection by using the integrated device. CMOS-compatible SiNWs were fabricated by anisotropic selfstop etching and PDMS chips were fabricated by rapid, simple, and cost-effective fabrication techniques. A leakage-free bond was formed in several minutes between the SiNWs and the PDMS chips. Capillary action was induced by the hydrophilicity of the channels using PVP. The simple and rapid multiplexed electrical detection of miRNA-126 and Cyfra21-1 was presented with high sensitivity and selectivity, showing great promise for point-of-care disease diagnostics.

\section{Acknowledgments}

We thank the National Key Research and Development Program of China (Nos. 2017YFB0405400 and 2017YFA0207103), the Project of National Natural Science Foundation of China (Nos. 91323304, 61327811, and 91623106), and the Project for Shanghai Outstanding Academic Leaders (No. 15XD1504300) for financial support.

\section{References}

1 G. F. Zheng, F. Patolsky, Y. Cui, W. U. Wang, and C. M. Lieber: Nat. Biotechnol. 23 (2005) 1294.

2 W. U. Wang, C. Chen, K. H. Lin, Y. Fang, and C. M. Lieber: Proc. Natl. Acad. Sci. U.S.A. 102 (2005) 3208.

3 M. Ferrari: Nat. Rev. Cancer 5 (2005) 161.

4 M. M. C. Cheng, G. Cuda, Y. L. Bunimovich, M. Gaspari, J. R. Heath, H. D. Hill, C. A. Mirkin, A. J. Nijdam, R. Terracciano, T. Thundat, and M. Ferrari: Curr. Opin. Chem. Biol. 10 (2006) 11.

5 K. I. Chen, B. R. Li, and Y. T. Chen: Nano Today 6 (2011) 131.

6 A. R. Gao, N. Lu, Y. C. Wang, P. F. Dai, T. Li, X. L. Gao, Y. L. Wang, and C. H. Fan: Nano Lett. 12 (2012) 5262. 
7 E. Stern, J. F. Klemic, D. A. Routenberg, P. N. Wyrembak, D. B. Turner-Evans, A. D. Hamilton, D. A. LaVan, T. M. Fahmy, and M. A. Reed: Nature 445 (2007) 519.

8 I. Park, Z. Y. Li, A. P. Pisano, and R. S. Williams: Nanotechnology 21 (2010) 015501.

9 S. Rasappa, D. Borah, C. C. Faulkner, T. Lutz, M. T. Shaw, J. D. Holmes, and M. A. Morris: Nanotechnology 24 (2013) 65503.

10 N. Lu, A. R. Gao, P. F. Dai, H. J. Mao, X. L. Zuo, C. H. Fan, Y. L. Wang, and T. Li: Anal. Chem. 87 (2015) 11203.

11 F. Zhou, Z. Y. Li, Z. T. Bao, K. Feng, Y. Zhang, and T. Wang: Scand. J. Clin. Lab. Invest. 75 (2015) 578.

12 M. W. Toepke and D. J. Beebe: Lab Chip 6 (2006) 1484.

13 I. Wong and C. M. Ho: Microfluid. Nanofluid. 7 (2009) 291.

14 A. R. Gao, N. Lu, P. F. Dai, T. Li, H. Pei, X. L. Gao, Y. B. Gong, Y. L. Wang, and C. H. Fan: Nano Lett. 11 (2011) 3974.

15 Y. L. Wang, X. X. Li, T. Li, H. Yang, and J. W. Jiao: IEEE Sens. J. 6 (2006) 686.

16 J. H. Chua, R. E. Chee, A. Agarwal, S. M. Wong, and G. J. Zhang: Anal. Chem. 81 (2009) 6266.

17 B. R. Huang, Y. K. Yang, and H. L. Cheng: Nanotechnology 24 (2013) 475502.

18 M. Chen, C. J. Hou, D. Huo, M. Yang, and H. B. Fa: Anal. Methods 7 (2015) 9466.

19 S. Mizuguchi, N. Nishiyama, T. Iwata, T. Nishida, N. Izumi, T. Tsukioka, K. Inoue, T. Uenishi, and S. Suehiro: Lung Cancer 58 (2007) 369.

20 M. Crawford, E. Brawner, K. Batte, L. Yu, M. G. Hunter, G. A. Otterson, G. Nuovo, C. B. Marsh, and S. P. Nana-Sinkam: Biochem. Biophys. Res. Commun. 373 (2008) 607.

21 M. J. Garnett, E. J. Edelman, S. J. Heidorn, C. D. Greenman, A. Dastur, K. W. Lau, P. Greninger, I. R. Thompson, X. Luo, J. Soares, Q. S. Liu, F. Iorio, D. Surdez, L. Chen, R. J. Milano, G. R. Bignell, A. T. Tam, H. Davies, J. A. Stevenson, S. Barthorpe, S. R. Lutz, F. Kogera, K. Lawrence, A. McLaren-Douglas, X. Mitropoulos, T. Mironenko, H. Thi, L. Richardson, W. J. Zhou, F. Jewitt, T. H. Zhang, P. O'Brien, J. L. Boisvert, S. Price, W. Hur, W. J. Yang, X. M. Deng, A. Butler, H. G. Choi, J. Chang, J. Baselga, I. Stamenkovic, J. A. Engelman, S. V. Sharma, O. Delattre, J. Saez-Rodriguez, N. S. Gray, J. Settleman, P. A. Futreal, D. A. Haber, M. R. Stratton, S. Ramaswamy, U. McDermott, and C. H. Benes: Nature 483 (2012) 570.

22 A. He, T. C. Liu, Z. N. Dong, Z. Q. Ren, J. Y. Hou, M. Li, and Y. S. Wu: J. Clin. Lab. Anal. 27 (2013) 277.

\section{About the Authors}

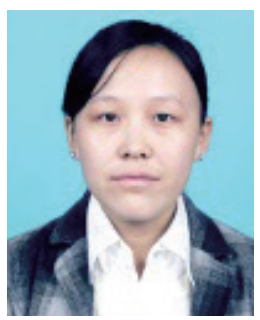

Anran Gao received her B.S. degree from Qingdao University in 2008 and her Ph.D. degree from Shanghai Institute of Microsystem and Information Technology, Chinese Academy of Sciences, in 2013. Her research interests include the design, analysis, and fabrication of semiconductor devices, and finding their applications in biochemical sensing.

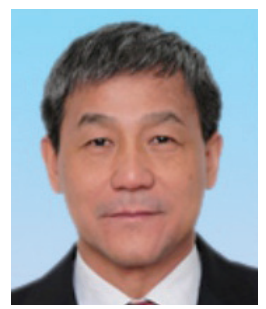

Yuelin Wang received his B.S. degree from Zhejiang University in 1982, his M.S. degree from Harbin Institute of Technology in 1985, and his Ph.D. degree from Tsinghua University in 1989. He is currently a professor at Shanghai Institute of Microsystem and Information Technology. His research interests include micro-electromechanical and nano-electromechanical systems.

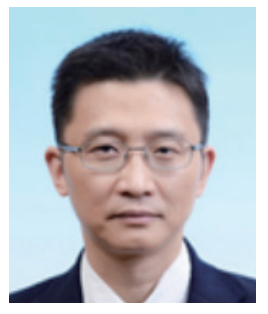

Tie Li received his B.S. and Ph.D. degrees in condensed matter physics from the University of Science and Technology of China, Hefei, in 1992 and 1997, respectively. He is currently a professor in the MEMS Lab at Shanghai Institute of Microsystem and Information Technology. His research interests include the mechanism, fabrication, and application of micro-nanofused sensors. 